\title{
On a class of nonlinear fourth order differential equations. $\left(^{*}\right)$
}

\author{
by PUI-KeI WoNe (**)
}

Summary. - In this paper we study the nonlinear fourth order differentiai equation $u^{i v} \pm$ $F^{\prime}(u, v) u=0$, where $F^{\prime}$ is a pssitive monotone function of $u$. Asymptotic behaviour of certain solutions are treated in sections 2 and 4 nhile a two point boundary value problem is studied in section 3 .

1. - In this paper we are concerned with nonlinear differential equations of the form

$$
u^{i v}-F(u, x) u=0
$$

and

$$
u^{i v}+F(u, x) u=0,
$$

where $u^{i v}=d^{4} u / d x^{4}$. The function $F$ is assumed to satisfy the following hypotheses:

H1. $F(u, x)$ is continuous in $u$ and $x$ for $x \geq x_{0}$ and $0 \leq u<\infty$,

H2. $F(u, x)>0$ for $u>0$ and $x \geq x_{0}$,

H3. $U^{-2 \varepsilon} F(u, x)$ is a strictly increasing function of $u$ for each $x \geq x_{0}$ and some constant $\varepsilon>0$.

We remark that many of the results remain true if $H 3$ is replaced by the weaker assumption that $F(u, x)<F(v, x)$ for $0<u<v<\infty$ and $x \geq x_{0}$, i.e., with $\varepsilon=0$ in H3. It will be apparent in the proofs whether the weaker hypothesis will suffice.

In sections 2 and 4 of this paper we present some necessary and sufficient conditions for solutions of equations (1.1) and (1.2) respectively to have prescribed asymptotic forms. In section 3 a two point boundary value problem for equation (1.1) is treated by a method used on second order equations [7]. For related studies on the oscillatory behavior of solutions of

see $[2,3,5]$.

$$
y^{(n)}+f(x) g(y)=0
$$

(*) Entrata in Redazione il 26 agosto 1968.

(**) Michigan State University, East Lansing, Michigan, U.S.A. 
2. - In this section we shall study the asymptotic behavior of some positive proper solutions of equation (1.1). By a proper solution we understand a real-valued solution of (1.1) which is of class $C^{4}[a, \infty)$, where $a \geq x_{0}$. Analogous results have been obtained earlier [8] for the corresponding second order equation $u^{\prime \prime}=F(u, x) u$. Actually, it will be apparent from our method of proof that these same results may be extended to the equation $u^{(n)}=F(u, x) u$.

To facilitate discassion wo shall call a proper solution of (1.1) a solution of type I if $(-1)^{k} u^{(k)}(x) \geq 0, k=0,1,2,3$. A proper solution $u$ is of type II if $u$ is eventually positive, convex increasing, and $u^{\prime \prime \prime}$ is of one sign.

Theonem $2.1-A$ necessary and sufficient condition for equation (1.1) to have proper solutions $u$ of type $I$ which ultimately decrease monotonically to dositive constants is that

$$
\int^{\infty} x^{3} F(B, x) d x<+\infty
$$

for some constant $B>0$.

Proof. - If $u$ is such a solution, then by TAYlon's Theorem,

$$
u(x)=\sum_{k=0}^{3}(-1)^{k} u^{(k)}(b)(b-x)^{k} / k !+(1 / 6) \int_{x}^{b}(t-x)^{3} u F(u, t) d t
$$

Since $(-1)^{k} u^{(k)}(x) \geq 0$ for $k=0,1,2,3$, it follows that

$$
\begin{aligned}
u(a) & \geq u(x) \geq(1 / 6) \int_{x}^{b}(t-x)^{3} u F(u, t) d t \\
& \geq(C / 6) \int_{x}^{b}(t-x)^{3} F(C, t) d t,
\end{aligned}
$$

where $C=\lim u(x)>0$. (2.1) follows from this immediately.

To prove sufficiency, we consider the integral equation

$$
u(x)=C+(1 / 6) \int_{*}^{\infty}(t-x)^{3} u(t) F(u, t) d t
$$


Suppose $B$ is a positive constant for which (2.1) holds, then there must be a point $a \geq x_{0}$ such that for all $x \geq a$,

$$
\int_{x}^{\infty}(t-x)^{3} F(B, t) d t<3
$$

We now define an approximating sequence $\left\{\boldsymbol{u}_{k}(x)\right\}$ by

$$
\left\{\begin{array}{l}
u_{0}(x)=C, \\
u_{k+1}(x)=O+(1 / 6) \int_{x}^{\infty}(t-x)^{3} u_{k} F\left(u_{k}, t\right) d t \\
k=0,1,2, \ldots
\end{array}\right.
$$

If we choose $C$ such that $0<C<B / 2$, we see immediately that, for all $k, 0<C \leq u_{k}(x)$. By assuming $u_{k}(x)<B$ and using (2.4), we find that

$$
u_{k+1}(x)<(B / 2)+(B / 6) \int_{x}^{\infty}(t-x)^{s} F(B, t) d t<B .
$$

Since $u_{0}(x)<B$, it follows by induction that $0<C \leq u_{k}(x)<B$ for all $k$. Moreover, for any two points $x_{1}$ and $x_{2}$ such that $a \leq x_{1}<x_{2}<\infty$, a simple calculation shows that

$$
\text { 2) } u_{n+1}\left(x_{2}\right)-u_{n+1}\left(x_{1}\right)|\leq| x_{2}-x_{1} \mid \int_{x_{1}}^{\infty}\left(t-x_{1}\right)^{2} u_{n}\left(t \cdot F^{\prime}\left(u_{n}, t\right) d t\right.
$$

In view of the uniform boundedness of $\left\{\boldsymbol{u}_{n}\right\}$ and condition $(2.1)$, it follows that the sequence is equicontinuous also. According to $\mathrm{H} 3, F(u, x)<$ $<F(v, x)$ whenever $0<u<v<\infty$, it follows from the assumption $u_{n+1}(t) \geq$ $\geq u_{n}(t)$ and (2.5) that

$$
u_{n+2}(x)-u_{n+1}(x)=\int_{x}^{\infty}(t-x)^{3}\left[u_{n+1} F\left(u_{n+1}, t\right)-u_{n} F\left(u_{n}, t\right)\right] d t>0
$$

This, together, with the fact that $u_{1}(x)>u_{0}(x)$, shows that $\left\{u_{k}(x)\right\}$ is also a monotonically increasing sequence. We can therefore extract a uniformly 
convergent subsequence whose limit function $u$ is a solution of $(2.3)$ in $[a, \infty)$.

It remains to show that the solution $u$ so obtained is indeed a proper solution of type $\mathrm{I}$ which satisfies (1.1). To this end we note that for $x \geq a$ and $h>0$,

$$
\begin{gathered}
\left|h^{-1}[u(x+h)-u(x)]+(1 / 2) \int_{x}^{\infty}(t-x)^{2} F(u, t) u(t) d t\right| \\
\leq(h / 6)\left\{\int_{x+\hbar}^{\infty}|3(t-x)-h| F(u, t) u(t) d t+\int_{x}^{x+h}|t-x-3 h| u F(u, l) d t\right\} .
\end{gathered}
$$

A corresponding inequality holds for $h<0$. Since the solution $u$ is continuous in $[a, \infty)$, it follows that

$$
2 u^{\prime}(x)=-\int_{x}^{\infty}(t-x)^{2} u(t) F(u, t) d t \leq 0 .
$$

In a similar manner one can show successively that

$$
\begin{aligned}
& u^{\prime \prime}(x)=\int_{x}^{\infty}(t-x) u(t) F(u, t) d t \geq 0, \\
& u^{\prime \prime \prime}(x)=-\int_{x}^{\infty} u(t) F(u, t) d t \leq 0,
\end{aligned}
$$

and $u^{i v}=u F(u, x)$. This proves the theorem.

Before proceeding to the next theorem on solutions of type II, we first state as a lemma the following well known (see e.g., [1]). Tauberian theorem:

Lemma 2.2. - Let $f(x) \sim x^{s}, s>0$. If $f^{\prime}(x)$ is positive, integrable and mo. notonically non-decreasing, then $f^{\prime}(x) \sim s x^{s-1}$.

Theorem 2.3. - A necessary and sufficient condition for equation (1.1) to have positive proper solutions of type II with asymptotic form

$$
u(x) \sim C x^{m}, \quad C>0, m=1,2,3
$$

is that there exist a constant $B>0$ such that

$$
\int_{x}^{\infty} x^{m+3} F\left(B x^{m}, x\right) d x=0\left(x^{m}\right)
$$

for all sufficiently large $x$. 
Proof. - If $u$ is such a solution, then (2.2) shows that

$$
\begin{gathered}
x^{-m} u(x) \geq x^{-m}\left[u^{\prime}(b)(x-b)+u^{\prime \prime \prime}(b)(x-b)^{3} / 3 !\right] \\
+\left(1 / 6 x^{m}\right) \int_{x}^{\infty}(t-x)^{3} u(t) F(u, t) d t .
\end{gathered}
$$

In view of (2.2) there exists a point $a \geq x_{0}$ such that

$$
1 / 2 \leq u(x) / C x^{m} \leq(3 / 2)
$$

for all $x \geq a$. Moreover, for $m=1,2,3$, Lemma 2.2 shows successively that the quantity

$$
\left|x^{-m}\left[u^{\prime}(b)(x-b)+u^{\prime \prime}(b)(x-b)^{3} / 6\right]\right|
$$

is bounded nuiformly so that

$$
3 C / 2+M \geq\left(C / 12 x^{m}\right) \int_{x}^{b}(t-x)^{3} t^{m} F\left(C t^{m} / 2, t\right) d t
$$

for all $b>x \geq a$. The necessity of (2.7) follows immediately.

As in the proof of Theorem 2.1, the sufficiency of condition (2.7) depends on our solving the integral equation

$$
v(x)=C+\left(1 / 6 x^{m}\right) \int_{i}^{\infty}(t-x)^{3} t^{m} v(t) F\left(t^{m} v, t\right) d t
$$

by iteration. Except for the obvious computational differences, the arguments involved are entirely analogous and will therefore be omitted. If $v(x)$ is the solution of (2.8) so obtained, the function $u(x)=x^{m} v(x)$ will be the desired solution of (1.1) having asymptotic form (2.6).

3. - In this section we shall consider a two-point boundary value prob. lem associated with equation (1.1), namely,

$$
\begin{aligned}
& u^{i v}-u F(u, x)=0 \\
& u(\alpha)=u^{\prime}(a)=u(b)=u^{\prime}(b)=0,
\end{aligned}
$$

where $x_{0} \leq a<b<\infty$. With suitable modifications, the methods used here can be applied to the problem with a triple zero at $a$ and a simple zero at $b$. 
In earlier papers Mooke and NeHaRI [6] and NeHari [7] have studied analogous problems for the equations $u^{\prime \prime}+p(x) u^{2 n+1}=0$ and $u^{\prime \prime}+u F\left(u^{2}, x\right)=0$ respectively.

Problem (3.1) is equivalent to a variational problem for which the direct methods of the calculus of variations are applicable. The results given here are direct extensions of those obtained by NEHART in [7].

We begin with the functional $J$ defined by

$$
\left.\int_{a}^{\infty}\left[u^{\prime \prime}\right)^{2}-2 h(u, x)\right] d x
$$

where

$$
h(u, x)=\int_{0}^{u} t F(t, x) d t .
$$

It is easy to see that the EULER-Lagrange equation associated with the functional $J$ is precisely equation (1.1). Let $\Omega$ denote the family of twice differentiable function on $[a, b]$ for which $u(a)=u^{\prime}(a)=0, u(x) \geq 0$ on $(a, b)$ aLd $u(x) \equiv \equiv \equiv$. Moreover, we further suppose that $u$ satfsfies the normalization condition

$$
\int_{a}^{b}\left(\boldsymbol{u}^{\prime \prime}\right)^{2} d x=\int_{a}^{b} \boldsymbol{u}^{2} F(u, x) d x .
$$

If $v$ is any function of $\Omega$ then $\mathrm{H} 3$ implies the existence of a positive constant $k$ such that $u=k v$ will satisfy (3.4) We can now state the principal result of this section as follows:

THeOREM 3.1. - The variational problem

$$
\min _{\Omega} J(u)=\lambda(a, b)
$$

has a solution $u \in O^{4}[a, b]$ such that $u$ is also a solution of problem (3.1).

Proof. - Since we are concerned with a mininum problem we may further restrict ourselves to those functions of $\Omega$ for which

$$
\int_{a}^{b}\left(u^{\prime \prime}\right)^{2} d x \leq M,
$$

where $M$ is some fixed positive constant. Let $\Omega^{*}$ denote this subset of $\Omega$. 
By H3, $u>t>0$ implies $u^{-2 \varepsilon} F(u, x)>t^{-2 \varepsilon} F(t, x)$ so that

$$
\begin{aligned}
h(u, x) & =\int_{0}^{u} t F(t, x) d t \leq u^{-2 \varepsilon} F(u, x) \int_{0}^{u} t^{1+2 \varepsilon} d t \\
& =u^{2} F(u, x) /(2+2 \varepsilon) .
\end{aligned}
$$

It follows that

$$
u^{2} F(u, x)-2 h(u, x) \geq u^{2} F(u, x) /(1+\varepsilon)
$$

Combining this with condition (3.4) we see that

$$
J(u) \geq(1+\varepsilon)^{-1} \int_{a}^{b}\left(u^{\prime \prime}\right)^{2} d x>0 .
$$

Hence, $\lambda(a, b)=\inf _{\Omega^{*}} J(u)$ exists and the variational problem (3.5) is therefore meanigful.

According to TAYLon's theorem, we have

$$
u(x)=\int_{a}^{x}(x-t) u^{\prime \prime}(t) d t
$$

Applying SoHwarz's inequality and (3.6) we arrive at the estimate

$$
|u(x)|^{2} \leq M(b-a)^{3} / 3, \quad x \in[a, b]
$$

Moreover, for any two points $x_{1}$ and $x_{2}$ such that $a \leq x_{1}<x_{2} \leq b$,

$$
\begin{aligned}
\left|u\left(x_{2}\right)-u\left(x_{1}\right)\right| & \leq\left|x_{2}-x_{1}\right| \int_{a}^{x_{2}}\left|u^{\prime \prime}(t)\right| d t+\int_{x_{1}}^{x_{2}}\left|x_{1}-t\right|\left|u^{\prime \prime}(t)\right| d t \\
& \leq\left|x_{2}-x_{1}\right|[2 M(b-a)]^{1 / 2}
\end{aligned}
$$

so that the family $\Omega^{*}$ of admissible function is in fact an uniformly bounded and equi-continuous family. Consequently, there exists a sequence $\left\{u_{n}\right\}$ in $\Omega^{*}$ which converges uniformly on $[a, b]$ to a continuous function $u$ such that

$$
\lim J\left(u_{n}\right)=J(u)=\lambda(a, b)
$$


Let $u$ be any member of the minimizing sequence given above. We now construct a new function $y$ of class $C^{4}[a, b]$ by solving the linear inhomogeneous system

$$
\begin{aligned}
& y^{i v}=a u F(u, x) \\
& y(a)=y^{\prime}(a)=y(b)=y^{\prime}(b)=0,
\end{aligned}
$$

where the constant $\alpha$ is determined by requiring $y$ to satisfy condition (3.4). Integration by parts shows that

$$
\int_{a}^{b} u y^{i v} d x=\int_{a}^{b} u^{\prime \prime} y^{\prime \prime} d x
$$

Combining this with $(34)$ and $(3.10)$ we have

$$
\begin{aligned}
{\left[\left.\alpha \int_{a}^{b} u^{2} F(u, x) d x\right|^{2}\right.} & =\left|\int_{a}^{b} u^{\prime \prime} y^{\prime \prime} d x\right|^{2} \leq \int_{a}^{b}\left(u^{\prime \prime}\right)^{2} d x \int_{a}^{b}\left(y^{\prime \prime}\right)^{2} d x \\
& =\int_{a}^{b} u^{2} F(u, x) d x \int_{a}^{b} y^{2} F(y, x) d x
\end{aligned}
$$

Consequently,

$$
\alpha^{2} \int_{a}^{b} u^{2} F(u, x) d x \leq \int_{a}^{b} y^{2} F(y, x) d x
$$

On the other hand,

$$
\begin{aligned}
{\left[\left.\int_{a}^{b} y^{2} F(y, x) d x\right|^{2}\right.} & \left.=\mid \int_{a}^{b}\left(y^{\prime \prime}\right)^{2} d x\right]^{2}=\left[\left.\int_{a}^{b} y^{i v} y d x\right|^{2}\right. \\
& =\alpha^{2}\left[\left.\int_{a}^{b} y u F(u, x) d x\right|^{2}\right. \\
& \leq \alpha^{2} \int_{a}^{b} u^{2} F(u, x) d x \int_{a}^{b} y^{2} F(u, x, d x
\end{aligned}
$$

so that, by $(3.12)$, we have

$$
\int_{a}^{b} y^{2} F(y, x) d x \leq \int_{a}^{b} y^{2} F(u, x) d x .
$$


Now $F(y, x)$ is an increasing function of $y$ so that (3.3) implies

$$
2[h(u, x)-h(y, x)] \leq F(u, x)\left(u^{2}-y^{2}\right)
$$

Combining this with (3.4) and (3.13) we finally arrive at

$$
J(u) \geq J(y)
$$

This argument shows that the minimizing sequence $\left\{u_{n}\right\}$ may be replaced by another minimizing sequence $\left\{y_{n}\right\}$ which is of class $C^{4}[a, b]$. In view of (3.6) and (3.12), we have

$$
\alpha_{n}^{2} \lambda(a, b) \leq M
$$

Moreover, we know that

$$
y_{n}(x)=\alpha_{n} \int_{a}^{b} g(x, t) u_{n}(t) F\left(u_{n}, t\right) d t
$$

where $g(x, t)$ is the Grees's function for the linear operator

$$
\begin{aligned}
& L u=-u^{i v} \\
& u(a)=u^{\prime}(a)=u(b)=u^{\prime}(b)=0 .
\end{aligned}
$$

It follows from the uniform congergence of $\left\{u_{n}\right\}$ that the sequence $\left\{y_{n}\right\}$ will necessarily converge to a function $y \in O^{4}[a, b]$. Moreover, in view of (3.14) and (3.5), we have

$$
\lambda=\lim J\left(u_{n}\right) \geq \lim J\left(y_{n}\right)=J(y)=\lambda
$$

so that

$$
J(y)=\lambda(a, b)=\frac{\inf }{\Omega^{*}} J(u) .
$$

We now show that $y(x) \neq 0$ on $[a, b]$. To do this we set

$$
A^{2}=\int_{a}^{b}\left(y^{\prime \prime}\right)^{2} d x
$$

so that

$$
\left[y^{\prime}(x)\right]^{2}=\left(\int_{a}^{x} y^{\prime \prime}(t) d t\right)^{2} \leq A^{2}(x-a) \leq A^{2}(b-a)
$$




$$
\begin{aligned}
y(x)^{2} & =\left(\int_{a}^{x} y^{\prime}(t) d t\right)^{2} \leq A^{2}(b-a)(x-a)^{2} \\
& =B^{2}(x-a)^{2}
\end{aligned}
$$

where $B^{2}=A^{2}(b-a)$. Thus, $y(x) \leq B(x-a)$. Combining this with (3.4) and (3.15) we arrive at

$$
1 \leq(b-a) \int_{\alpha}^{b}(x-a) F[B(x-a), x] d x
$$

In view of the monotonicity hypothesis H3, this implies $A$ is bounded from below by $A_{0}$. Consequently, we have

$$
\int_{a}^{b}\left(y^{\prime \prime}\right)^{2} d x \geq A_{0}^{2}>0
$$

From this we conclude that $y(x) \equiv \equiv$.

Finally we note that equality can hold in (3.14) only if equality holds in (3.13) which in turn requires equality in ScHwARz's inequality, i.e., $y=u$ on $[a, b]$. In this case $y$ must in fact be a solution of

$$
\begin{aligned}
& y^{i v}=\alpha y F(y, x) \\
& y(a)=y^{\prime}(a)=y(b)=y^{\prime}(b)=0
\end{aligned}
$$

Integration by parts show that

$$
\int_{a}^{b}\left(y^{\prime \prime}\right)^{2} d x=\alpha \int_{a}^{b} y^{2} F(y, x) d x
$$

Comparing this with normalization (3.4) we conclude that $\alpha=1$. Thus, equality is attained in (3.14) only if $y$ is a solution of problem (3.5). This completes the proof of the theorem.

Our next result shows the effect on the minimum value $\lambda(a, b)$ when the fnnction $F$ is altered. Before we state this theorem we first note that if $a^{\prime} \leq a<b \leq b^{\prime}$, then it follows trivially that

$$
\lambda\left(a^{\prime}, b^{\prime}\right) \leq \lambda(a, b)
$$


TheoRem 3.2. - Let $F_{1}$ and $F_{2}$ be two functions satisfying hypotheses H1-3. Moreover, we suppose that for each $t>0$ and $x \in[a, b], F_{1}(l, x) \leq F_{2}$ $(t, x)$. If $\lambda_{1}(a, b)$ and $\lambda_{2}(a, b)$ are the respective minima associated with the equations

$$
\begin{aligned}
& u_{k}^{i n}=u_{k} F_{k}^{\prime}\left(u_{k}, x\right), \\
& u_{k}(a)=u_{k}^{\prime}(a)=u_{k}(b)=u_{k}^{\prime}(b)=0, \quad k=1,2,
\end{aligned}
$$

then $\lambda_{1}(a, b) \geq \lambda_{2}(a, b)$.

Proof. - Let $u_{1}$ be a solution of the system (3.17) associated with the function $F_{1}$. Then by the monotonicity property of $F_{2}$ we can find a positive constant $A$ such that

$$
\int_{a}^{b}\left(u_{1}^{\prime \prime}\right)^{2} d x=\int_{a}^{b} u_{1}^{2}, F_{2}\left(A u_{1}, x\right) d x
$$

The function $w(x)=A u_{1}(x)$ will elearly satisfy the normalization

$$
\int_{a}^{b}\left(w^{\prime \prime}\right)^{2} d x=\int_{a}^{b} w^{2} F_{2}(w, x) d x
$$

and is therefore a member of the admissible family $\Omega_{2}$, It follows that

$$
\lambda_{2}(a, b)=\min _{\Omega_{2}} J_{2}(u) \leq \int_{a}^{b}\left[\left(w^{\prime \prime}\right)^{2}-2 h_{2}(w, x)\right] d x,
$$

Since $F_{2}(t, x) \geq F_{1}(t, x),(3.3)$ shows that $h_{2}(w, x) \geq h_{1}(w, x)$. Moreover,

$$
h_{1}\left(A u_{1}, x\right)-h_{1}\left(u_{1}, x\right)=\int_{u_{1}}^{A u_{1}} t F_{1}(t, x) d t \geq \frac{1}{2}\left(A^{2}-1\right) u_{1}^{2} F_{1}\left(u_{1}, x\right)
$$

so that

$$
2 \int_{a}^{b} h_{1}\left(A u_{1}, x\right) d x \geq 2 \int_{a}^{b} h_{1}\left(u_{1}, x\right) d x+\left(A^{2}-1\right) \int_{a}^{b} u_{1}^{2} F_{1}\left(u_{1}, x\right) d x .
$$


These three inequalities together with normalization (3.4) for $\overrightarrow{u_{1}}$ show that

$$
\begin{aligned}
\lambda_{2}(a, b) & \leq \int_{a}^{b}\left[\left(w^{\prime \prime}\right)^{2}-2 h_{1}(w, x)\right] d x \\
& =A^{2} \int_{a}^{b}\left(u_{1}^{\prime \prime}\right)^{2} d x-2 \int_{a}^{b} h_{1}\left(A u_{1}, x\right) d x \\
& \leq A^{2} \int_{a}^{b}\left(u_{1}^{\prime \prime}\right)^{2} d x-2 \int_{a}^{b} h_{1}\left(u_{1}, x\right) d x+\left(1-A^{2}\right) \int_{a}^{b} u_{1}^{2} F_{1}\left(u_{1}, x\right) d x \\
& =\int_{a}^{b}\left[\left(u_{1}^{\prime \prime}\right)^{2}-2 h_{1}^{-}\left(u_{1}, x\right)\right] d x \\
& =\lambda_{1}(a, b) .
\end{aligned}
$$

This proves our assertion.

4. - In this section we shall treat briefly some asymptotic properties of positive solutions of equation (1.2). Oscillation criteria for equations of this type have been given recently [2] and [3]. Theorem 4.4 given here is an ex. tension of a result of LEIGHTON and NEHARI [4] for linear fourth-order equations.

Lemma 4.1. - Let $p(x)$ be positive and continuous for $x \geq x_{0}$. Then no nontrivial solution of the differential inequality

$$
w^{i v}+p(x) w \leq 0
$$

can have more than one double zero on $[a, \infty)$, where $a \geq x_{0}$.

Proof. - Let $w$ be a nontrivial solution of (4.1), and we set

$$
G(x)=w^{\prime} w^{\prime \prime}-w w^{\prime \prime} .
$$

Then,

so that, for $b>a$,

$$
\begin{aligned}
G^{\prime}(\boldsymbol{x}) & =\left(w^{\prime \prime}\right)^{2}-w w^{i v} \\
& \leq\left(\boldsymbol{w}^{\prime \prime}\right)^{2}+p(\boldsymbol{x}) w^{2}>0
\end{aligned}
$$

$$
G(b)-G(a)=\int_{a}^{b} G^{\prime}(x) d x>0 .
$$


If $w$ has a double zero ot a point $c \in[\alpha, \infty)$, then (4.2) shows that $G(c)=0$. By (4.3), $G$ is a strictly increasing function of $x$ for $x>c$ so that $G$ cannot vanish for $x>c$. This proves the assertion.

Lmma 4.2. - The boundary value problem

$$
\left\{\begin{array}{l}
u^{i v}+F(u, x) u=0 \\
u(a)=A, \quad u(b)=B \\
u^{\prime}(a)=A^{\prime}, u^{\prime}(b)=B^{\prime}
\end{array}\right.
$$

with $b>a$ and $A, B>0$ has at most one positive solution in $[a, b]$.

Proof. - Suppose the contrary. Let $u$ and $y$ be two positive solutions of (4.4). We further assume that $(a, A)$ and $(b, B)$ are consecutive points of intersection of $u$ and $y$. Set $u(x)>y(x)$ for $a<x<b$, then

$$
(u-y)^{i v}+u F(u, x)-y F(y, x)=0
$$

or

$$
(u-y)^{i v}+(u-y) F(y, x)+u[F(u, x)-F(y, x)]=0
$$

Now, $u(x)>y(x)$ implies $F(u, x)>F(y, x)$ so that, by setting $w(x)=u(x)-y(x)$, we obtain

$$
w^{i v}+w F(y, x) \leq 0
$$

Since both $u$ and $y$ are solutions of (4.4), $w(x)$ clearly has double zeros at $a$ and $b$ which is impossible as Lemma (4.1) is applicable to inequality (4.5). This being true for each consecutive pairs of points of intersection, the result follows.

Lemma 4.3. - Let $u$ be a solution of (1.2) such that $u(x)>0$ for $x>a$. Then

$$
\lim _{x \rightarrow \infty} 6 x^{-3} u(x)=\lim _{x \rightarrow \infty} u^{\prime \prime \prime}(x)
$$

Proof. - Let $P_{3}(x)$ be a polynomial of degree 3, then every solution $u$ of equation (1.2) can be written as

$$
6 P_{3}(x)=6 u(x)+\int_{a}^{x}(x-t)^{3} u(t) F(u, t) d t
$$


Hence,

$$
\begin{aligned}
6 P_{3}(x) & \leq 6 u(x)+(x-a)^{3} \int_{a}^{x} u(t) F(u, t) d t \\
& =6 u(x)+(x-a)^{3}\left[u^{\prime \prime \prime}(a)-u^{\prime \prime \prime}(x)\right] .
\end{aligned}
$$

Now, it is clear that

$$
\lim _{x \rightarrow \infty}(x-a)^{3} P_{3}(x)=u^{\prime \prime \prime}(a)
$$

and that $u^{\prime \prime \prime}(x)$ is a monotonically decreasing function as $x$ tends to $\propto$ so that

$$
\lim u^{\prime \prime \prime}(x) \leq \lim \text { inf } 6 u(x)(x-a)^{-3} .
$$

On the other hand, by the mean value theorem, we can find a point $s \in(a, x)$ such that

$$
\begin{aligned}
6 P_{3}(x) & \geq 6 u(x)+\int_{a}^{s}(x-t)^{3} u(t) F(u, t) d t \\
& \geq 6 u(x)+(x-s)^{3} \int_{a}^{s} u(t) F(u, t) d t \\
& \geq 6 u(x)+(x-s)^{3}\left[u^{\prime \prime \prime}(a)-u^{\prime \prime \prime}(s)\right] .
\end{aligned}
$$

Fixing $s$, we find that

$$
6(x-a)^{-3} P_{3}(x) \geq(x-a)^{-3} 6 u(x)+\left[u^{\prime \prime \prime}(a)-u^{\prime \prime \prime}(s)\right][(x-s) /(x-a)]^{-3} .
$$

By taking $x$ through a sequence of points $\left\{x_{n}\right\}$ such that $(x-a)^{-3} u(x)$ tends to its upper limit, we see that for $s>a$.

$$
u^{\prime \prime \prime}(s) \geq \lim \sup (x-a)^{-3} 6 u(x)
$$

so that

$$
\lim \sup 6(x-a)^{-3} u(x) \leq \lim u^{\prime \prime \prime}(s)
$$

Combining (4.7) with (4.8) we obtain the desired result.

Theorem 4.4. - A necessary and sufficient condition for equation (1.2) to have a positive solution $u$ such that

$$
u(x) \sim A x^{3}, \quad A>0
$$


is that there exists a constant $B>0$ for which

$$
\int^{\infty} x^{3} F\left(B x^{3}, x\right) d x<+\infty
$$

Proof. - To prove sufficiency we suppose that (4.10) is satisfied for some $B>0$. We can then find a point a large enough so that

$$
\int_{a}^{\infty} x^{3} F\left(B x^{3}, x\right) d x<5
$$

Consider the solution $u$ given by

$$
6 B(x-a)^{3}=6 u(x)+\int_{a}^{x}(x-t)^{3} u(t) F(u, t) d t
$$

If we can find a point $b>a$ such that, for $a<x<b, u(x)>0$ and $u(b)=0$, then

$$
B x^{3} \geq B(x-a)^{3} \geq u(x) \quad x \in(a, b) .
$$

Now,

$$
\begin{aligned}
6 B b-a)^{3} & =6 u(b)+\int_{a}^{b}(b-t)^{3} u(t) F(u, t) d t \\
& =\int_{a}^{b}(b-t)^{3} u(t) F(u, t) d t \\
& \leq(b-a)^{3} \int_{a}^{b} u(t) F(u, t) d t
\end{aligned}
$$

so that by $(4.11)$,

$$
6 B \leq \int_{a}^{b} u(t) F(u, t) d t \leq B \int_{a}^{b} x^{3} F\left(B x^{3}, x\right) d x<5 B
$$

which is clearly absurd. We therefore conclude that $u(x) \neq 0$ for $x \in(a, \infty)$, i.e., $u(x)$ is nonoseillatory. 
With the help of the Volterra equation equivalent to (1.2), we see from (4.12) that for $a \leq x<+\infty$,

$$
6 B(x-a)^{3} \leq 6 u(x)+B(x-a)^{3} \int_{a}^{x}(t-a)^{3} F\left(B t^{3}, t\right) d t .
$$

Combining this with (4.11) and (4.12), we have

$$
B(x-a)^{3} \leq 6 u(x) \leq 6 B(x-a)^{3} .
$$

In view of Lemma 4.3 , this establishes the sufficiency.

To prove necessity, we let $u(x)$ be a positive solution of (1.2) such that (4.9) holds. Then

$$
\int_{a}^{\infty} u(x) F(u, x) d x=u^{\prime \prime \prime}(a)-6 A
$$

Now, $u(x) \geq 0$ implies $u^{i v}(x) \leq 0$ so that $u(x) \geq(A-\varepsilon) x^{3}$ for some $\varepsilon>0$.

Hence,

$$
u^{\prime \prime \prime}(a) \geq(A-\varepsilon) \int_{a}^{\infty} x^{3} F\left[(A-\varepsilon) x^{3}, x\right] d x
$$

This completes the proof of the theorem.

\section{REFERENCES}

[1] G. H. HARDY, Divergent Series, Oxford University Press, 1949, London.

[2] H. C. How ARD, Oscillation criteria for even ordev differential equations, Ann. Mat. Pura Appl., 66 (1964), pp. 221-231.

[3] I. T. KIGURADZE, Oscillation properties of solutions of certain ordinary differential equa. tions, Dokl. Nauk SSSR, 144 (1962), pp. 33-36.

[4] WALTER LeIGHTON and ZEEV NEHARI, On the oscillation of solutions of self-adjoint linear differential equations of the fourth order, Trans. Amer. Math. Soc, 89 (1958), pp. $325-377$.

[5] I. Licko and M. SVEc, Le caractere oscillatoire des solutions de l'equation $y^{(n)}+$ $+f(x) y^{\alpha}=0, n>1$, Czech. Math. J., 13 (1968), 481.491.

[6] R. A. MOORE and ZEEV NEHARI, Nonoscillation theorems for a class of nonlinear diffe. rential equations, Trans. Amer. Math. Soc. 93 (1959), pp. 30.52.

[7] Znev NeHARI, On a class of nonlinear second-order differential equations, Trans. Amer. Math. Soc., 95 (1960), pp. 101-123.

[8] PUI-KEI WONG, Existence and asymptotic behavior of proper solutions of a class of second-order nonlinear differential equations, Pacific J. Math., 13 (1963), pp. 737.760. 